\title{
CONTRIBUTION OF THE CORTICO-CLAUSTRAL LOOP TO RECEPTIVE FIELD PROPERTIES IN AREA 17 OF THE CAT ${ }^{1}$
}

\author{
HELEN SHERK ${ }^{2}$ AND SIMON LEVAY \\ Department of Neurobiology, Harvard Medical School, Boston, Massachusetts 02115
}

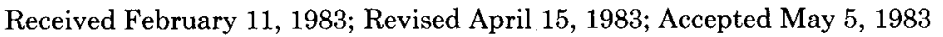

\begin{abstract}
The contribution of the cat's claustrum to the response properties of cells in area 17 was studied by destroying the left claustrum and examining receptive field properties of cells in both the left and right area 17. For each cell we assessed sharpness of orientation tuning, degree of direction selectivity, length summation, end-stopping, ocular dominance, responsiveness, and several other properties. On the lesioned side, 462 cells were studied, and on the control side, 636 cells.

All cats showed a reduction in the number of end-stopped cells in area 17 on the side of the lesion. This was particularly marked in layers $2+3$ and 4 , where end-stopped cells are normally most abundant. In the control hemisphere, $43 \%$ of the sample from these layers showed at least moderate end-stopping whereas, in contrast, only $21 \%$ exhibited this degree of end-stopping on the side of the lesion. There was no obvious change in other response properties. We conclude that one function of the cat's claustrum is to help regulate the length selectivity of cells in area 17.
\end{abstract}

Of the various brain structures that project to area 17 , only the lateral geniculate nucleus (LGN) provides an input whose function is known; the contributions made by other structures are as yet unclear. The dorsocaudal (visual) claustrum is one such structure. It receives its visual input from several visual cortical areas, including area 17, and projects back to the same areas (Jayaraman and Updyke, 1979; Sanides and Buchholtz, 1979; Carey et al., 1980; Olson and Graybiel, 1980; Squatrito et al., 1980; LeVay and Sherk, 1981a); both limbs of this loop are retinotopically organized (Olson and Graybiel, 1980; LeVay and Sherk, 1981b). Claustral axons distribute to all cortical layers, but most heavily to those receiving the bulk of the geniculate input, layers 4 and 6 (Carey et al., 1980; LeVay and Sherk, 1981a). It is the function of this pathway that forms the topic of the present study.

In a previous study (Sherk and LaVay, 1981) we have described the response properties of neurons in the cat's visual claustrum. Virtually all cells in this region respond to appropriate visual stimuli. Like cells in several other visual structures, claustral cells are binocular and strongly prefer moving to stationary stimuli. They lack any directional selectivity. Perhaps uniquely among subcortical neurons in the cat, claustral cells are orientation

\footnotetext{
${ }^{1}$ We thank Torsten Wiesel for comments on the manuscript and Doris Ringer for secretarial help. This work was supported by National Institutes of Health Grant EY-R01-1960 to S. L.

${ }^{2}$ Present address: Department of Biological Structure, University of Washington, School of Medicine, Seattle, WA 98195.
}

selective, with a degree of specificity approaching that found in area 17. The most striking property of claustral cells, however, is their length summation: short slits or edges are generally poor stimuli, but their responses improve as the stimulus is extended, often up to 30 or $40^{\circ}$.

Whether claustro-cortical axons are excitatory or inhibitory is not definitely known, although some evidence from electrical stimulation experiments suggests that they are excitatory (Tsumoto and Suda, 1982). If excitatory, however, they seem unlikely to form a dominant input to many cells of the visual cortex since, outside of layer 6, cortical neurons generally display response properties rather different from those common in the claustrum. It is more likely that the claustrum exerts a predominantly inhibitory effect on the visual cortex, either directly or by way of cortical interneurons. We reasoned that if this were the case, the claustrum might contribute to the selectivity of cortical cells for stimulus properties such as direction of movement or length.

To investigate the influence of the claustrum on area 17 , we destroyed one visual claustrum and compared response properties of cells in area 17 on the lesioned side with those in the intact hemisphere. The lesion method has distinct limitations but still serves as a useful starting point for an investigation of function. We looked for changes in cortical response properties following loss of claustral input, for changes in the proportion of cell types encountered, and for changes associated with particular laminae. 


\section{Materials and Methods}

Most of the methods used in preparation and recording have been described previously (LeVay and Sherk, 1981a). Cats were initially anesthetized with ketamine injected intramuscularly; anesthesia was then maintained either with intravenous pentobarbital or (in two cats) with ketamine. The cat was intubated and placed in a stereotaxic frame, and a craniotomy was made over the left claustrum. The animal was then paralyzed with gallamine triethiodide and ventilated at a rate yielding end-tidal $\mathrm{CO}_{2}$ levels between 3.3 and $4 \%$. Plano contact lenses focused the eyes at 57 inches $(145 \mathrm{~cm})$. Focus was judged, and retinal landmarks were plotted, by the use of a fiberoptic illuminator (Pettigrew et al., 1979).

The left visual claustrum was first located with a tungsten microelectrode. As noted previously (Sherk and LeVay, 1981), there is some inter-animal variability in the vigor and reliability of visual responsiveness in the claustrum. If we considered responses to be insufficiently brisk or reliable, we ended the experiment at this point. Otherwise, the electrode was replaced with a pipette containing $0.2 \%$ kainic acid, through which visual responses were also recorded in order to ensure accurate placement of the tip within the claustrum. In most experiments $0.5 \mu \mathrm{l}$ of kainic acid was injected over a period of 30 to $60 \mathrm{~min}$ in order to destroy the visual claustrum; in early experiments larger volumes $(1.6 \mu \mathrm{l})$ were used.

In four cats recordings were then made in the ipsilateral (left) area 17 until about $30 \mathrm{hr}$ had elapsed from the start of the experiment. In the other four cats the contralateral (right) area 17 was studied in this first recording session. The infusion of the paralytic agent was then stopped and the cat was resuscitated; it usually slept for at least $24 \mathrm{hr}$ thereafter. Two animals died during resuscitation and thus gave data only from the right, control area 17.

Five to 8 days later, a second recording session was carried out. The procedure was identical except that we did not record from the claustrum, and we studied area 17 contralateral to the side investigated in the first session.

We tried to make the cortical penetrations as similar as possible on the two sides. The electrode was placed in a parasagittal plane and tilted either anterior or posterior about $40^{\circ}$ from vertical. To maximize the time spent in layers $2+3$ and 4 , the electrode was generally angled down the medial bank close to the cortical surface. Because the claustrum projects most densely to relatively peripheral area 17 (LeVay and Sherk, 1981b), and because there is also a weak crossed projection from the contralateral claustrum to the region of the area 17-18 border (LeVay and Sherk, 1981a), we did not begin collecting data until receptive fields were found to be at least $5^{\circ}$ away from the vertical meridian. From this point on, we studied every cell isolated until the electrode passed out of the brain, or we ended the experiment. On average, 77 cells were studied in area 17 on the side of the lesion (range, 56 to 104) and 79 cells on the control side (range, 56 to 117 ).

We assessed a standard list of properties for each cell. These were: optimal orientation, degree of orientation selectivity, length summation, end-stopping, optimal stimulus width (perpendicular to preferred orientation), edge preference (light or dark), ocular dominance, receptive field size and location, responsiveness, and spontaneous activity. For every parameter except field size and location, each cell's response was subjectively assigned to one of a small number of categories. For example, endstopping was categorized as total, strong, medium, weak, or absent, and ocular dominance was categorized according to the seven-point scale of Hubel and Wiesel (1962). Cells were also categorized by type. They were considered to be simple if they had separate on- and off-responsive subfields (Hubel and Wiesel, 1962); standard complex if their on and off responses were mixed and they clearly summated up to the field length; special complex if on and off responses were mixed and the cell gave a brisk response to a short stimulus and summated very little, or not at all, up to the field length (Gilbert, 1977); e-on if they gave brisk on responses and no off responses (Toyama and Takeda, 1974); e-off if they showed the converse behavior; and unresponsive if we could not elicit sufficient response to plot a receptive field. For statistical purposes we grouped the e-on and e-off cells with simple cells.

A small electrolytic lesion was made at the end of each penetration, and usually two more were placed at 1- or $1.5-\mathrm{mm}$ intervals as the electrode was withdrawn. These allowed cells to be assigned to cortical layers with a reasonable degree of accuracy when the penetration was reconstructed histologically. The inaccuracy introduced by making lesions during electrode withdrawal was offset by the fact that the electrode usually stayed in each layer for a considerable distance, due to the angle of the penetration.

Cats were perfused with formol/saline after the second (or, in one case, third) recording session. Area 17 was sectioned parasagittally on a Vibratome and stained with cresyl violet. The claustrum and front end of the LGN were cut coronally to determine the extent of the lesion.

\section{Results}

In all cats the kainic acid injections caused complete destruction of the visual claustrum. One of the smaller lesions is shown in Figure 1. The ventral, nonvisual stem of the nucleus was partially spared, but the visual region showed a total loss of neurons. Although in this case the damage was almost entirely confined to the claustrum, in the early experiments, in which larger injection volumes were used, the lesions encroached on the putamen and adjacent cortex. In one cat the extreme rostral tip of the LGN was also affected.

We studied 462 cells in area 17 on the side of the lesion (six cats) and 636 cells on the control sde (eight cats). In all six cats for which data were available from both hemispheres, there was a reduction in end-stopping on the side of the lesion. We could detect no changes in other receptive field properties.

Normally, end-stopped cells are not found with equal frequency in all layers. They are relatively numerous in layer $2+3$, for example, whereas layer 6 has been reported to have almost none (Gilbert, 1977; Ferster, 1981; but see Kato et al., 1978). We thus concentrated on the upper 


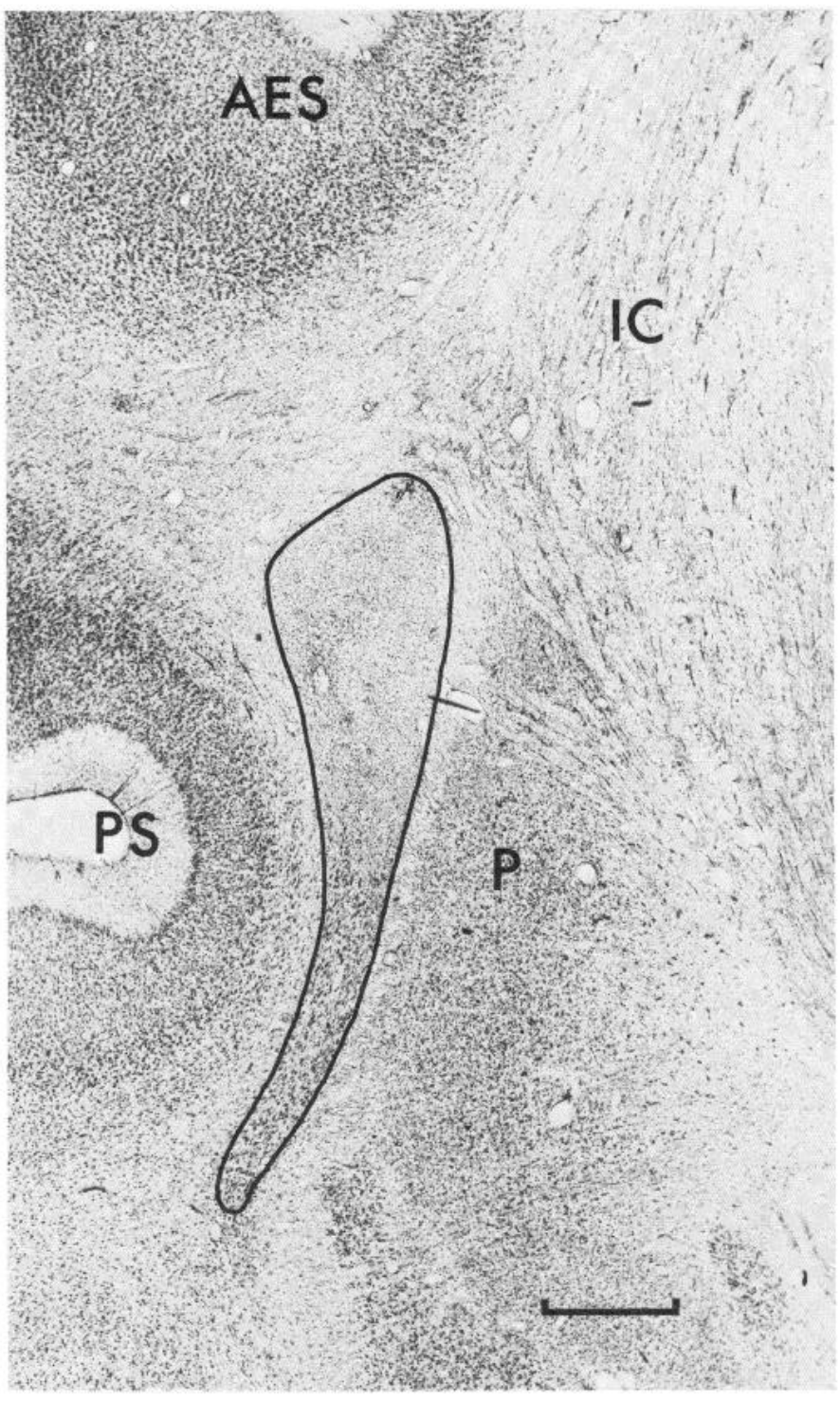

Figure 1. A Nissl-stained section through the center of a lesion in the left claustrum, produced by the injection of $0.5 \mu \mathrm{l}$ of $0.2 \%$ kainic acid. There are no surviving neurons in the visual claustrum; the remaining cells are glia. Part of the ventral, nonvisual stem of the claustrum remains intact. $A E S$, anterior ectosylvian sulcus; $I C$, internal capsule; $P$, putamen; $P S$, pseudosylvian sulcus. The scale marker is $1 \mathrm{~mm}$.

layers, $2+3$ and 4 , where end-stopped cells are comparatively abundant, after early experiments indicated that claustral lesions might affect end-stopping. The heavy claustral input to layer 4 also suggested that effects might be most obvious here.

We analyzed the reduction of end-stopping separately for each layer. For every cell, end-stopping was assessed as total (no response to a long slit), strong, medium, weak, or absent. The results from layers $2+3$ and 4 , for all animals combined, are tabulated in histogram form in Figure 2. In both these layers, there was a marked reduction in the proportion of cells that showed medium, strong, or total end-stopping (groups 2, 3, and 4 in Fig. 2 ). In layer $2+3$, cells in these three categories comprised $28 \%$ of the experimental sample, compared to $49 \%$ of the control sample. On the lesioned side, there was a corre- sponding increase in the proportion of cells that showed no end-stopping. The decline was still more marked in layer 4, with only $13 \%$ of the experimental sample showing at least moderate end-stopping compared to $37 \%$ on the control side. The differences were significant in both these layers $\left(p<0.001, \chi^{2}\right.$ test).

End-stopping was also reduced in layer 5, with 13 of 69 cells on the experimental side showing at least medium end-stopping compared to 26 of 78 cells on the control side. We consider these samples too small, however, to permit firm conclusions regarding the effect of claustral lesions on this layer. In layer 6 , we found virtually no end-stopped cells on either side.

There was no reduction in the proportion of cells
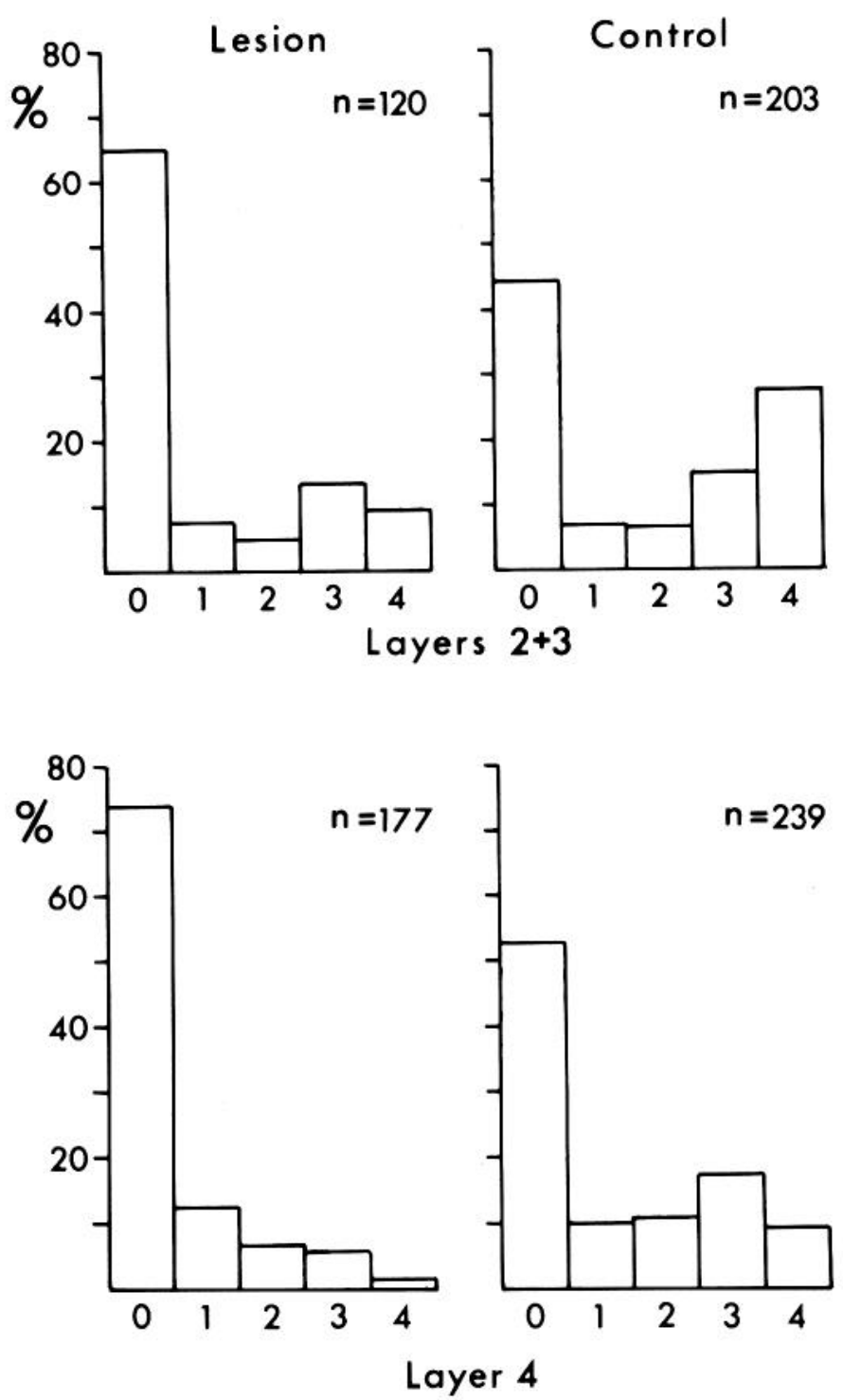

Figure 2. Percentages of cells in each end-stopping category for layers $2+3$ (upper histogram) and layer 4 (lower histogram), pooled from all cats. Cells in group 0 had no end-stopping; in group 1 , end-stopping was weak; in group 2 , it was medium, in group 3, it was strong; and in group 4, end-stopping was total. The left histogram of each pair shows data taken from the lesioned hemisphere, and the right histogram shows data from the control hemisphere. The fraction of cells in groups 2 to 4 was reduced on the lesioned side for both layers. 
classified as weakly end-stopped. This might have been because cells that originally displayed stronger end-stopping lost most of this as a consequence of claustral ablation and were then classed as weakly end-stopped. Furthermore, because we included in this class cells whose end-stopping was variable or ambiguous, we may have counted some cells that actually had none.

To determine whether complex and simple cells were equally affected by lesioning the claustrum, we have divided our samples according to cell type, lumping together cells from all layers. These are shown in Figure 3. Standard and special complex cells were grouped together, since there was no obvious difference between them. Nor was there a great difference between simple and complex cells, although the former showed a somewhat greater reduction in end-stopping. For both cell
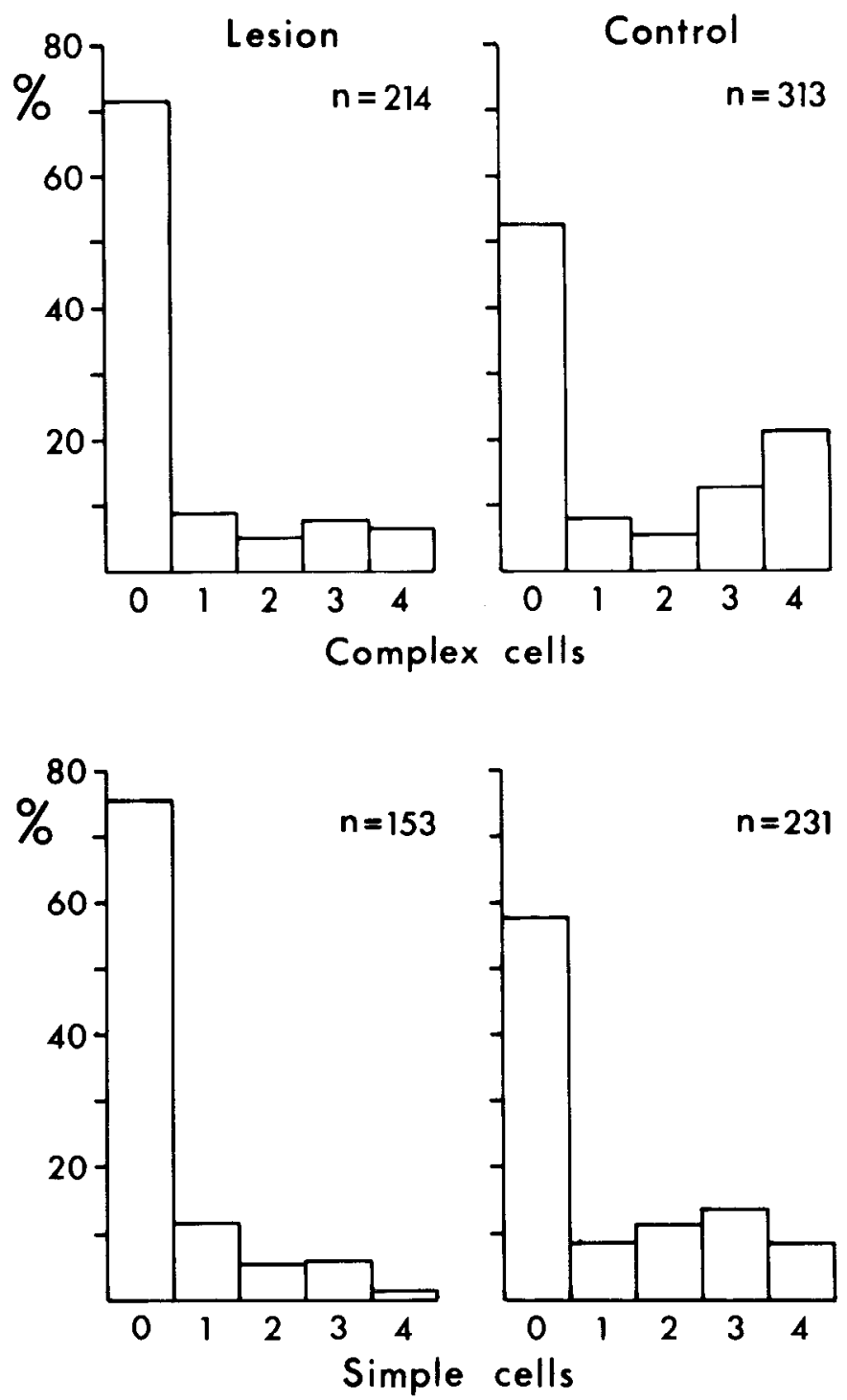

Figure 3. Percentages of cells in each end-stopping category for complex cells (upper histograms) and simple cells (lower histograms), pooling data for all cats and all layers. Categories are the same as for Figure 2. There were fewer moderately, strongly, or totally end-stopped cells on the lesioned side (left histograms) than on the control side (right histograms) for both types of cells.

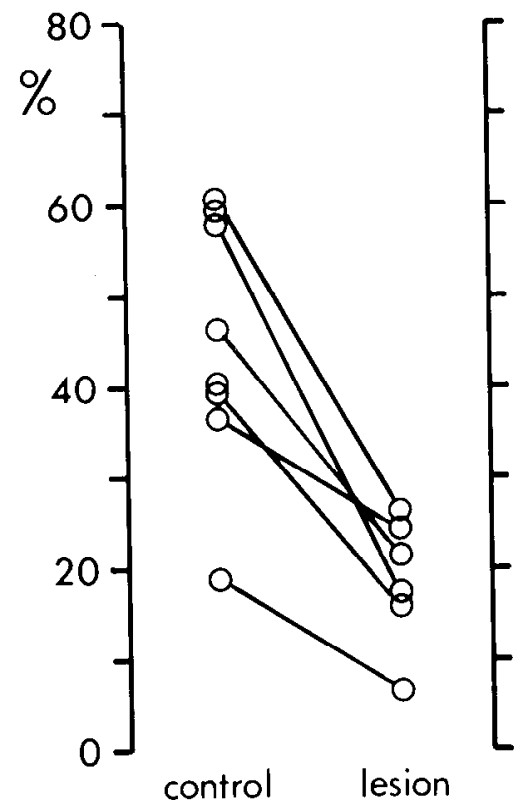

Figure 4. Percentages of cells from layers $2+3$ and 4 that were at least moderately end-stopped, plotted for each cat separately. Each left-hand circle indicates data for one cat from the control side and is connected to the value for the same animal from the lesioned side. The two cats for which only control data were obtained are represented by left-hand circles only. All cats in which both sides were studied showed a decline in the fraction of end-stopped cells.

types, the loss of end-stopping was statistically significant $\left(p<0.001, \chi^{2}\right.$ test).

The changes that we saw suggested that some endstopped cells either lost their end-stopping when deprived of claustral input or became unresponsive. In either case, one might ask whether these cells differed in some fashion from those end-stopped cells that were unaffected. As Figure 3 shows, cell type did not seem to be a major distinguishing factor. Other response properties that we tested (listed under "Materials and Methods") were also similar for end-stopped cells on the lesioned and control sides. There remains the possibility that the end-stopping that survived on the lesioned side was of the asymmetric type, i.e., more pronounced at one end of the cell's receptive field, but we did not test for this.

As mentioned above, end-stopping was reduced in every cat. Figure 4 shows, for each cat separately, the proportion of cells in the upper layers $(2+3$ and 4$)$ that had medium, strong, or total end-stopping. The probability that the differences between the lesioned and control hemispheres were due to chance is only 0.002 (onetailed, unpaired Wilcoxon test).

It is evident from Figure 4 that there was some cat-tocat variation in the amount of end-stopping lost. The reduction was about the same in area 17 whether it was studied immediately after the claustral lesion or some days later; thus, the time elapsed after destruction of the claustrum did not seem to be a critical factor. The major variability was in the proportion of end-stopped cells found in the control hemispheres; the range on this side was about twice that on the lesioned side (see Fig. 4). It 
is not clear what caused this. Although there may be some intrinsic variability, we suspect that other poorly controlled factors such as anesthetic level were the main source. If, as our results indicate, the claustrum contributes to the end-stopping seen in the cortex, the variation may reflect the variability of responsiveness in the claustrum itself. This tends to be greater from one animal to another than is the case for area 17 (Sherk and LeVay, 1981). It is possible that cats showing little end-stopping on the control side had a relatively depressed claustrum on that side. We tried to exclude such animals by using only ones in which we could find vigorous claustral responses, but there is no guarantee that these remained vigorous, particularly during the second recording session.

In addition to end-stopping, other response properties were examined. These included orientation preference, degree of orientation selectivity, degree of directional preference, length summation, width preference, edge preference (light or dark), field location and size, ocular dominance, velocity preference, responsiveness, and spontaneous activity. As with end-stopping, for each of these other properties we assigned cell responses to one of several categories (e.g., direction selectivity was assessed as total, strong, medium, weak, or absent). For none of these properties was there a clear difference between the samples from the lesioned and control hemispheres. Nor were the proportions of different cell types altered (the types we considered were simple, standard complex, special complex, e-on, e-off, and unresponsive; the criteria for assigning cells to each class are described under "Materials and Methods"). Because of the limitations inherent in qualitative testing, we are not confident that all of these response properties actually remained normal, or that some subclass of cells was not specifically affected, perhaps rendered completely unresponsive. An increase in unresponsive cells might be particularly difficult to detect since such cells are generally missed unless they are spontaneously active. Nevertheless, it is clear that the most marked and consistent change was in end-stopping.

\section{Discussion}

The effect of claustral ablation was surprisingly specific. In every one of six cats, the number of end-stopped cells was reduced in area 17 on the side of the lesion compared to the contralateral, control side. Although, as discussed below, we hesitate to conclude that there were no other changes, this reduction in end-stopping was the only one that we could detect.

When Hubel and Wiesel (1965) first described endstopped ("hypercomplex") cells in the visual cortex, they suggested two alternative mechanisms by which this property might be generated. The first required the cell to receive inhibition from two other cells of similar orientation preference, with receptive fields situated one at each end of the excitatory field of the end-stopped cells, as in Figure 5A. In the second scheme the cell received inhibition from one other cell (again of similar orientation preference) whose receptive field was centered at the same position as its own, but which extended considerably further at either end, as shown in Figure
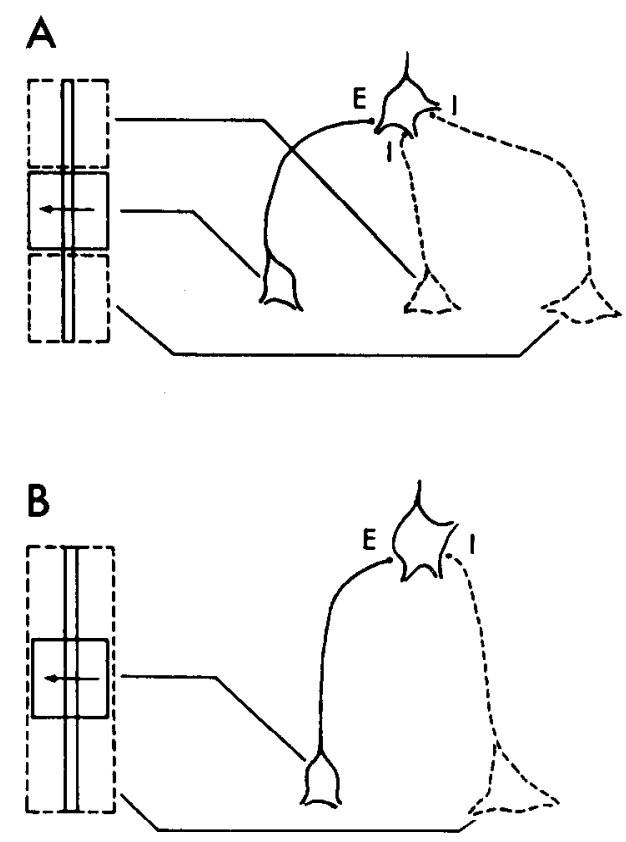

Figure 5. Wiring diagrams proposed by Hubel and Wiesel (1965) to account for the properties of end-stopped cells. $A$, The stopped cell receives excitatory input from a cell whose vertically oriented field is indicated by a solid rectangle; two additional cells inhibitory to the end-stopped cell have vertically oriented fields flanking the first one above and below, shown by dashed rectangles. In an alternative scheme $(B)$, the inhibitory input is supplied by a single cell with a large field, indicated by the entire dashed rectangle. In either case, a slit covering the entire field of the end-stopped cell would be ineffective. Scheme $B$ requires that a slit covering but restricted to the center region be too short to affect the inhibitory cell.

$5 B$. For this scheme to work, the inhibitory cell had to respond much better to long than to short slits. Sillito and Versiani (1977) have provided evidence in favor of the latter model: their results suggested that the inhibitory zone extended right through the central excitatory region, since stimulation of the central region was necessary for inhibition to be effective.

In several respects claustral cells are good candidates to provide this type of inhibition. They are orientation selective, respond much better to long than to short slits, yet respond poorly to a long slit with a central gap (Sherk and LeVay, 1981). They are relatively nonselective for aspects of the stimulus such as ocular dominance and direction of movement that are not relevant to the generation of length selectivity. They project to visual cortex and, as the present study has shown, their destruction results in a reduction, although not total elimination, of end-stopping in the cortex.

Several unanswered questions still remain. First, our results do not indicate whether the proposed inhibition is a direct effect of claustral cells on end-stopped cortical cells, or whether it involves excitation of a population of inhibitory cortical interneurons. According to Tsumoto and Suda (1982), who recorded the response of cortical neurons to electrical stimulation of the claustrum, the claustro-cortical projection is purely excitatory; inhibition was seen at longer latencies suggestive of a di- or a polysynaptic pathway. This implies that the claustrum 
influences most cortical cells by way of interneurons. In the present study we did not observe a loss of any cell population that might correspond to these interneurons, but such a loss could well have gone undetected, especially if the population were small.

It also remains to be established that claustral cells make connections with cortical cells having a similar orientation preference. However, such a mapping might well be facilitated by the orderly spatial layout of preferred orientations within the claustrum (Sherk and LeVay, 1981), combined with the columnar organization known to exist in the cortex (Hubel and Wiesel, 1962).

Another point to be resolved is whether the cells of claustrum operate over a range of stimulus lengths appropriate for generating the end-stopping seen in cortical neurons. In our sample from normal area 17, the average end-stopped cell began to show signs of inhibition to stimuli longer than $2^{\circ}$. The responses of some cells declined progressively as the stimulus length was increased to $40^{\circ}$ or more, whereas others were strongly inhibited by quite short stimuli. Although almost no claustral cells respond maximally to slits as short as $3^{\circ}$, some do respond well above background to slits this short (Sherk and LeVay, 1981). (Whether shorter slits are also effective is not known.) Thus some claustral cells may be able to exert the end-stopping seen in many small-field cortical cells, but if so they must produce effective inhibition at firing frequencies well below maximal. On the other hand, claustral cells could readily generate even the longest inhibitory end zones that we have observed, since they often summate to considerable lengths.

Because end-stopping, although reduced, was not abolished by claustral lesions, other circuits than the corticoclaustral loop must also be capable of generating this property. Of particular interest are the neurons in layer 6 of the visual cortex. In both area 17 (Gilbert, 1977) and area 18 (Ferster, 1981) these cells have strikingly elongated receptive fields and summate over the entire length of their fields without any end-stopping. In this respect they resemble-perhaps in a less extreme form-the receptive fields of claustral cells. Many layer 6 neurons (although not those projecting to the claustrum (LeVay and Sherk, 1981a)) have extensive collateral arborizations in layer 4. As Gilbert and Wiesel (1979) have suggested, this pathway might contribute to the endstopping seen in the upper layers and might thus be responsible for the end-stopping that survives claustral lesions. Another possibility is that the antagonistic surrounds of geniculate neurons make a contribution to the end-stopping of cortical cells. In this case, one might wonder why not all cortical cells are end-stopped. The answer might lie in the considerable variation in the strength of surround inhibition that is shown by different geniculate neurons (Bullier and Norton, 1979; J. C. Horton and H. Sherk, submitted for publication).

The existence of duplicate mechanisms for the generation of end-stopping is perplexing. Reliance on a distant nucleus such as the claustrum seems a peculiarly cumbersome means of achieving end-stopping if intracortical circuits are also capable of mediating this property. It is possible that the claustrum acts on a distinct subpopulation of end-stopped cells, although we were unable to find evidence that the remaining end-stopped cells differed from those in normal area 17. Alternatively, it may be that the modulation of the claustrum by its nonvisual inputs, from the lateral hypothalamus and the thalamic nucleus centralis, makes its contribution to end-stopping uniquely important.

We suspect, however, that the reduction in end-stopping that we have observed does not reveal the full extent of the influence exerted by the claustrum on area 17 . There could be changes to which our qualitative assessments were insensitive, such as subtle alterations in orientation selectivity or length summation. Or, some classes of cells might have become unresponsive-as long as such a class represented only a small fraction of the whole population, we would probably have overlooked its absence. Finally, a type of claustral function for which the lesion method cannot test is one involving coordination of activity between different visual cortical areas. Nevertheless, our results indicate that one function of the visual claustrum is to help regulate length selectivity in area 17.

\section{References}

Bullier, J. H., and T. T. Norton (1979) X and Y relay cells in cat lateral geniculate nucleus: Quantitative analysis of receptive-field properties and classification. J. Neurophysiol. 42: 244-273.

Carey, R. G., M. F. Bear, and I. T. Diamond (1980) The laminar organization of the reciprocal projections between the claustrum and striate cortex in the tree shrew, Tupaia glis. Brain Res. 184: 193-198.

Ferster, D. (1981) A comparison of binocular depth mechanisms in areas 17 and 18 of the cat visual cortex. J. Physiol. (Lond.) 311: 623-655.

Gilbert, C. D. (1977) Laminar differences in receptive field properties of cells in cat primary visual cortex. J. Physiol. (Lond.) 268: 391-421.

Gilbert, C. D., and T. N. Wiesel (1979) Morphology and intracortical projections of functionally characterized neurones in the cat visual cortex. Nature 280: 120-125.

Hubel, D. H., and T. N. Wiesel (1962) Receptive fields, binocular interaction and functional architecture in the cat's visual cortex. J. Physiol. (Lond.) 160: 106-154.

Hubel, D. H., and T. N. Wiesel (1965) Receptive fields and functional architecture in two nonstriate visual areas (18 and 19) of the cat. J. Neurophysiol. 28: 229-289.

Jayaraman, A., and B. V. Updyke (1979) Organization of visual cortical projections to the claustrum in the cat. Brain Res. 178: $107-115$.

Kato, H., P. O. Bishop, and G. A. Orban (1978) Hypercomplex and simple/complex cell classifications in cat striate cortex. J. Neurophysiol. 41: 1071-1095.

LeVay, S., and H. Sherk (1981a) The visual claustrum of the cat. I. Structure and connections. J. Neurosci. 1: 956-980.

LeVay, S., and H. Sherk (1981b) The visual claustrum of the cat. II. The visual field map. J. Neurosci. 1: 981-992.

Olson, C. R., and A. M. Graybiel (1980) Sensory maps in the claustrum of the cat. Nature 288: 479-481.

Pettigrew, J. D., M. L. Cooper, and G. G. Blasdel (1979) Improved use of tapetal reflection for eye-position monitoring. Invest. Ophthalmol. Vis. Sci. 18: 490-495.

Sanides, D., and C. S. Buchholtz (1979) Identification of the projection from the visual cortex to the claustrum by anter- 
ograde axonal transport in the cat. Exp. Brain Res. 34: 197200.

Sherk, H., and S. LeVay (1981) The visual claustrum of the cat. III. Receptive field properties. J. Neurosci. 1: 993-1002.

Sillito, A. M., and V. Versiani (1977) The contribution of excitatory and inhibitory inputs to the length preference of hypercomplex cells in layers II and III of the cat's striate cortex. J. Physiol. (Lond.) 273: 775-790.

Squatrito, S., P. P. Battaglini, C. Galletti, and E. Riva Sanseverino (1980) Autoradiographic evidence for projections from cortical visual areas $17,18,19$ and the Clare-Bishop area to the ipsilateral claustrum in the cat. Neurosci. Lett. 19: 265269.

Toyama, K., and T. Takeda (1974) A unique class of cat's visual cortical cells that exhibit either ON or OFF excitation for stationary light slits and are responsive to moving edge patterns. Brain Res. 73: 350-355.

Tsumoto, T., and K. Suda (1982) Effects of stimulation of the dorsocaudal claustrum on activities of striate cortex neurons in the cat. Brain Res. 240: 345-349. 